\title{
The association between six month intra- dialytic resistance training and muscle strength or physical performance in patients with maintenance hemodialysis: a multicenter retrospective observational study
}

Yoshifumi Moriyama ${ }^{1}$, Masahiko Hara ${ }^{2,3^{*}}$ D, Sae Aratani ${ }^{4}$, Hideaki Ishikawa ${ }^{5}$, Kenichi Kono ${ }^{6}$ and Masatake Tamaki ${ }^{2,7}$

\begin{abstract}
Background: Reduced muscle strength and physical performance are prevalent in patients of maintenance hemodialysis (MHD), and deleterious changes in these parameters are associated with increased mortality.

Methods: This retrospective observational study included 306 patients, who received a 6-month resistance exercise program during hemodialysis, three times per week on an outpatient basis. The training protocol consisted of two sets of 10 repetitions of knee extension, hip abduction, and hip flexion, using an elastic band in a sitting or supine position. Primary outcome measures included muscle strength, measured by percent knee extension muscle power to dry body weight (pKEMP-dBW), and physical performance, measured by short physical performance battery (SPPB). The adjusted mean differences in these variables during the 6 months were estimated using a multivariate linear regression model.

Results: The mean age with standard deviation was $70 \pm 11$ years. One hundred and sixty patients (52.3\%) were men and the dry weight was $55.6 \pm 11.3 \mathrm{~kg}$. Sarcopenia, defined as SPPB $\leq 8$, was present in $21.4 \%$ patients. Their hemodialysis adequacy was acceptable, with a Kt $/ \mathrm{N}$ of $1.65 \pm 0.29$, and their nutritional status was good, with a normalized protein catabolism rate of $0.89 \pm 0.18 \mathrm{~g} / \mathrm{kg} /$ day. During the 6 months, both pKEMP-dBW and SPPB showed a slight but significant increase with an adjusted mean difference of 2.8 (95\% confidence interval 1.3-4.3, $p<0.001)$ and $0.6(0.4-0.9, p<0.001)$, respectively.
\end{abstract}

Conclusions: Six-month resistance training was associated with improved muscle strength and physical performance in patients with MHD.

Keywords: Hemodialysis, Muscle strength, Physical performance, Quality of life, Resistance training

\footnotetext{
* Correspondence: hara@japanscr.org

${ }^{2}$ Department of Clinical Investigation, Japan Society of Clinical Research,

Kita-ku Umeda 1-11-1000, Osaka Ekimae 4th Bldg 10F, Osaka 530-0001, Japan

${ }^{3}$ Center for Community-based Healthcare Research and Education, Shimane

University, Izumo, Japan

Full list of author information is available at the end of the article
}

(c) The Author(s). 2019 Open Access This article is distributed under the terms of the Creative Commons Attribution 4.0 International License (http://creativecommons.org/licenses/by/4.0/), which permits unrestricted use, distribution, and reproduction in any medium, provided you give appropriate credit to the original author(s) and the source, provide a link to the Creative Commons license, and indicate if changes were made. The Creative Commons Public Domain Dedication waiver (http://creativecommons.org/publicdomain/zero/1.0/) applies to the data made available in this article, unless otherwise stated. 


\section{Background}

The number of patients with end-stage renal disease is increasing worldwide due to the increased prevalence of diabetic and hypertensive nephropathy with aging, resulting in an increased number of patients who receive maintenance hemodialysis (MHD) $[1,2]$. Since MHD is associated with poor quality of life (QOL) and survival, the identification of risk factors and appropriate interventional strategies for improving QOL and outcomes have been intensively investigated [3-5]. Following this, reduced muscle strength and physical performance is considered to be one of the most important common prognostic indices of low QOL and poor survival in patients on MHD [3-5].

In contrast, it is well known that physical exercise has a positive impact on muscle strength and physical performance in healthy elderly patients, regardless of the type of exercise [6]. In addition, many studies have assessed the benefits of strength training from bench to bedside; these include anabolic changes in skeletal muscle messenger ribonucleic acid, or in muscle insulin-like growth factor-I protein, muscle mass, sleep quality, psychiatric status, and quality of life [7-10]. Among the several forms of exercise training, intra-dialytic resistance training has been suggested as a potential strategy to correct and/or prevent reductions of muscle strength and physical performance in MHD patients, considering its convenience, adherence, and safety, with little influence on cardiovascular systems $[11,12]$. However, most studies evaluated the short-term impact on muscle strength or physical performance within 3 months by enrolling only a small number of patients $(<50)$, and little evidence is available regarding the association between resistance training during MHD and muscle strength or physical performance in a longer time period and with a larger number of patients [1318]. The purpose of this multicenter retrospective observational study was to evaluate the association between the 6-month intra-dialytic resistance training and serial changes in muscle strength and physical performance.

\section{Methods}

\section{Study patients}

This retrospective observational study included a total of 306 patients on MHD. MHD was provided at outpatient dialysis units three times per week, and they underwent an intra-dialytic resistance exercise program for 6 months between April 2012 and July 2016 at 18 Kaikokai group hospitals in Japan. Thus, we only included patients who basically adhered to, and were compliant with the 6-month training programs; we can take from this that our study has a significant selection bias. The data of patients on MHD from these 18 hospitals had been gathered and recorded in the database at Nagoya
Kyoritsu Hospital in a daily clinical practice and the study data presented in the tables were retrospectively extracted from this database as a secondary usage. The study protocol complied with the Helsinki Declaration standards and was approved by the Ethical Committee of Nagoya Kyoritsu Hospital (Approval No. K094-03). The requirement of written informed consent was waived as this study used retrospective data obtained from the aforementioned database. The patient's right to avoid enrollment was pledged in an opt-out fashion. Extracted data included patient backgrounds, laboratory data, $\mathrm{Kt} / \mathrm{V}$ as an index of hemodialysis adequacy (ideal value in the present study $\geq 1.4$ ), normalized protein catabolic rate (n-PCR) as an index of nutritional status (ideal range in the present study $0.9-1.4 \mathrm{~g} / \mathrm{kg} /$ day), and percent creatinine generation rate (\%CGR) as an index of muscle mass compared to healthy individuals (ideal value in the present study $\geq 100 \%$ ) [13-18]. Percent knee extension muscle power to dry body weight (pKEMP-dBW) was collected as representative values for the muscle strength of lower extremities. We evaluated physical performance using short physical performance battery (SPPB), where an SPPB $\leq 8$ was defined as the presence of sarcopenia [19]. The authors had full access to the data and take responsibility for its integrity.

\section{Resistance training and measurement of muscle strength} Resistance training was provided for 6 months, under the supervision of training therapists at first, then under a self-training manner during MHD three times per week on an outpatient basis. The training protocol consists of two sets of 10 repetitions of three types of resistance trainings of the lower extremities: knee extension, hip abduction, and hip flexion using an elastic band (TheraBand Resistance Band Loops, THERABAND, Ohio, USA) encircling both ankles or above the knees, in a sitting or supine position depending on patient preference or ability (Fig. 1). There were four colors of elastic bands and the resistance strength could be determined by the color. Resistance training was performed with slow movement, taking $8 \mathrm{~s}$ to complete each resistance training, consisting of $4 \mathrm{~s}$ eccentric and $4 \mathrm{~s}$ concentric movements. Hence, the total time of daily resistance training was 3 (kinds of training) * 10 (repetitions) *2 (sets) * $8 \mathrm{~s}=8 \mathrm{~min}$; this took approximately 15 min per day to complete, including preparations and intervals. We employed slow resistance training in our daily clinical practice because slow resistance training can be performed at a lower intensity, with little influence on the cardiovascular system [20]. Resistance strength was set based on the Borg rating of perceived exertion and rating 13 (somewhat hard) was set as the targeted strength [21]. The strength was re-evaluated in detail every 12 weeks in our daily clinical practice, 


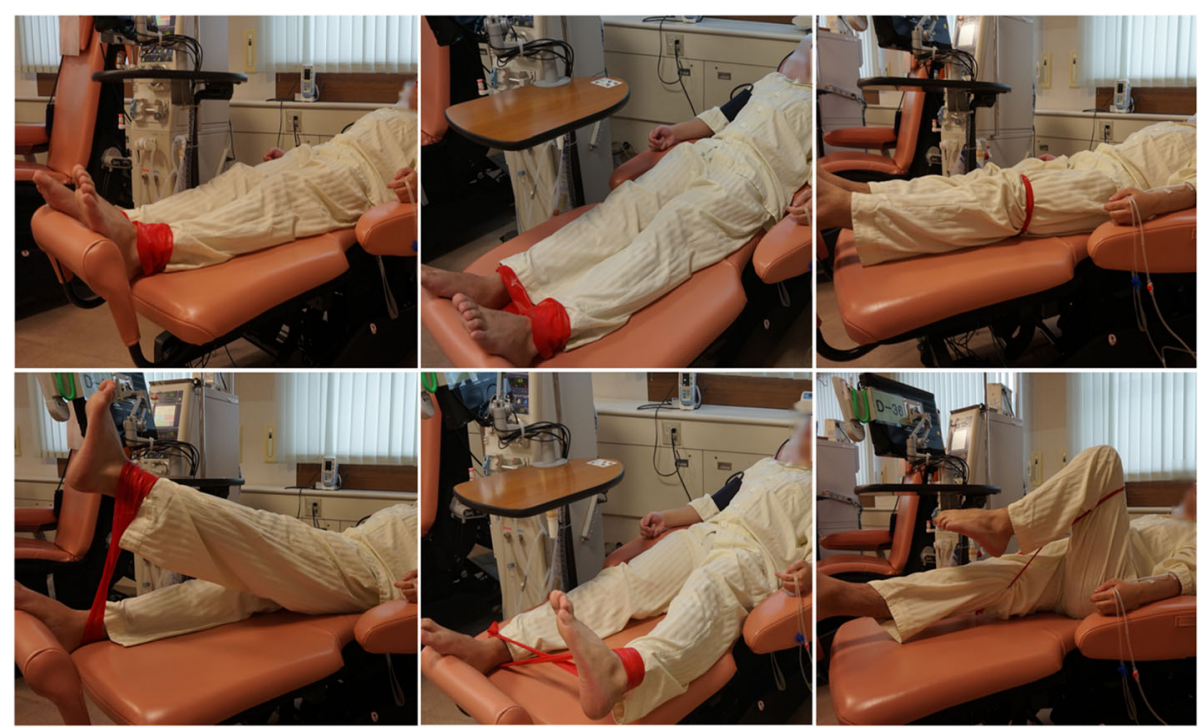

Fig. 1 Protocol of Resistance Training. Resistance training consists of two sets of 10 repetitions for knee extension (Left), hip abduction (Middle), and hip flexion (Right) using an elastic band (TheraBand Resistance Band Loops, THERABAND, Ohio, USA), encircling both ankles or above the knees in a sitting or supine position, depending on patient preference or ability. Resistance training was performed with slow movement, taking $8 \mathrm{~s}$ to complete each resistance training

whereas the resistance strength was adjusted at each training session based on the Borg rating as mentioned above. Thus, by selecting a color of the elastic band patients could select the resistance strength by themselves. Adverse events were defined as clinical events that occurred during intra-dialytic resistance training and required any kind of therapeutic intervention. Knee extension muscle strength was measured using a dynamometer ( $\mu$ Tas F-1 handheld dynamometer, Anima, Tokyo, Japan) at least twice before each MHD session, with the highest score being recorded. We used a handheld dynamometer because it was an easy to use, commercially available device widely used in Japan to measure knee strength [22, 23]. Although we could not source any validation data, the measurements were performed by an experienced therapist who was familiar with the device. Therefore, we believe that an accurate reading was obtained from this device. In addition, from a statistical viewpoint, because the comparison of serial changes was made using data from the same device, we do not expect the choice of device to affect the results.

\section{Endpoint and statistical analysis}

Primary outcome measures were set as serial changes in the average pKEMP-dBW of the right and left legs and the SPPB between the baseline and 6 months. The average pKEMP-dBW was calculated by the following formula: (highest pKEMP-dBW in right leg + highest pKEMP-dBW in left leg)/2. Secondary outcome measures were set as serial changes in pKEMP-dBW in each leg and 6 min walk distance. All values were measured before MHD. Continuous variables were summarized using the mean \pm standard deviation and categorical variables were summarized by percentages. The adjusted mean difference and 95\% confidence interval (CI) in the primary and secondary outcome measures during the 6 months were estimated using a multivariate linear regression model adjusted for age and sex. The significance level for our statistical analysis was set at 0.05 with the two-sided alternative hypothesis. If the Bonferroni correction was applied to the significance level to consider the problem of multiple comparisons, readers can also set the significance level at 0.01 . All statistical analyses were performed using the $\mathrm{R}$ software (version 3.4.2).

\section{Results}

Patient backgrounds are shown in Table 1. The mean age was $70 \pm 11$ years, $52.3 \%$ of the patients were men, and the dry weight was $55.6 \pm 11.3 \mathrm{~kg}$ with $159 \pm 8 \mathrm{~cm}$ body height. About half of the patients (44.2\%) reported that they had a habit of exercise. However, $24.4 \%$ patients were current smokers and $20.6 \%$ had a habit of alcohol consumption. Sarcopenia, defined as SPPB $\leq 8$, was present in $21.4 \%$ patients, with a \%CGR of $105 \pm 28$ at baseline. Their hemodialysis adequacy was acceptable, with a Kt/V of $1.65 \pm 0.29$, and their nutritional status was good, with an n-PCR of $0.89 \pm 0.18 \mathrm{~g} / \mathrm{kg} /$ day.

Regarding primary outcome measures shown in Table 2, both pKEMP-dBW and SPPB showed a slight but significant increase from $42.3 \pm 14.9 \%$ to $45.7 \pm$ 
Table 1 Patient Backgrounds

\begin{tabular}{ll}
\hline Parameter & $n=306$ \\
\hline Age, years & $70 \pm 11$ \\
Male & $52.3 \%$ \\
Height, cm & $159 \pm 8$ \\
Weight & \\
$\quad$ Set dry weight, kg & $55.6 \pm 11.3$ \\
$\quad$ Pre-HD weight, kg & $56.8 \pm 11.5$ \\
Exercise habit & $44.2 \%$ \\
Current smoker & $24.4 \%$ \\
Alcohol consumption habit & $20.6 \%$ \\
Laboratory data & \\
$\quad$ Creatinine, mg/dL & $10.2 \pm 2.6$ \\
Blood urea nitrogen, mg/dL & $61.3 \pm 15.7$ \\
Albumin, g/dL & $3.6 \pm 0.3$ \\
$\quad$ Hemoglobin, g/dL & $11.0 \pm 0.9$ \\
Total cholesterol, mg/dL & $157 \pm 33$ \\
Kt $N$ & $1.65 \pm 0.29$ \\
n-PCR, g/kg/day & $0.89 \pm 0.18$ \\
\%CGR & $105 \pm 28$ \\
Sarcopenia & $21.4 \%$ \\
\hline Continuous variables were summarized using the mean \pm standard deviation \\
and categorical variables were summarized by percentages \\
\%CGR percent creatinine generation rate, HD hemodialysis, $n-P C R$ & normalized \\
protein catabolic rate & \\
\end{tabular}

14.9\% (adjusted mean difference, 2.8; 95\% CI, 1.3-4.3, $p$ $<0.001)$ and from $10.0 \pm 2.6$ to $10.4 \pm 2.5(0.6,0.4-0.9$, $p<0.001$ ), respectively. In this study, the sample sizes required to detect the difference in primary outcome measures for muscle strength and physical performance were calculated to be 65 and 76, respectively, for a two-sided paired-t test with an alpha error of 0.05 and beta error of 0.80 . Secondary outcome measures showed similar results to those of the primary outcome measures, where right and left pKEMP-dBW increased significantly after 6 months (Table 2). In contrast, there was no statistical change in the 6 min walk distance $(p=0.988)$. There were no adverse events during the study period.

\section{Discussion}

In this multicenter retrospective observational study, we demonstrated that the 6-month resistance training program was associated with (1) increased muscle strength of the lower extremities, evaluated by pKEMP-dBW, and (2) increased physical performance, evaluated by SPPB in 306 patients with MHD. Considering that previous studies evaluated the impact of intra-dialytic resistance training from the data of a very limited number of patients (up to 79 before randomization, with the majority of studies providing a short-term 12-week training program), our data provide physicians with complementary insights in the resistance exercise training field for patients on MHD [13-18].

\section{Impact on muscle strength}

As all previous studies reported the improvement of muscle strength of lower extremities attributable to 3 to 6 months intra-dialytic resistance training, regardless of its intensity, our results are consistent with these reports [13-18]. For example, Cheema B, et al. demonstrated a statistically significant $15.2 \% \pm 15.4 \%$ improvement in total muscle strength associated with resistance training, whereas the control group showed a-2.4\% $\pm 13.8 \%$ change and no statistical significance [15]. This is consistent with our result of approximately $8 \%$ improvement in pKEMP-dBW. Since the improvement of muscle strength due to resistance training has also been reported in aging adults with or without diabetes, it is without a doubt that there is a favorable impact on muscle strength from resistance training [24-26]. In contrast to previous reports, there are two different points in the present study. That is, we provided resistance training in a self-training and slow movement manner. A recent randomized controlled trial $(n=296)$ of a 6-month personalized walking exercise program at home improved physical performance, including a 6-min walking test in patients on MHD [27]. Thus, we speculate that self-resistance training programs at home have the potential to be applicable for improving muscle strength in patients on MHD and this should be evaluated in a

Table 2 Serial Changes of Primary and Secondary Outcome Measures

\begin{tabular}{|c|c|c|c|c|}
\hline Parameter & Baseline & 6-Months Later & Difference $(95 \% \mathrm{Cl})^{\mathrm{a}}$ & $P$-value \\
\hline \multicolumn{5}{|l|}{ Primary Outcome } \\
\hline Average pKEMP-dBW, \% & $42.3 \pm 14.9$ & $45.7 \pm 14.9$ & $2.8(1.3-4.3)$ & $<0.001$ \\
\hline SPPB & $10.0 \pm 2.6$ & $10.4 \pm 2.5$ & $0.6(0.4-0.9)$ & $<0.001$ \\
\hline \multicolumn{5}{|l|}{ Secondary Outcome } \\
\hline Right pKEMP-dBW, \% & $42.6 \pm 15.2$ & $46.5 \pm 15.7$ & $3.6(1.9-5.3)$ & $<0.001$ \\
\hline Left pKEMP-dBW, \% & $41.9 \pm 15.5$ & $44.8 \pm 15.0$ & $2.1(0.5-3.7)$ & 0.012 \\
\hline Six minute walk distance, $\mathrm{m}$ & $324 \pm 175$ & $317 \pm 212$ & $0(-21-22)$ & 0.988 \\
\hline
\end{tabular}

Continuous variables were summarized using the mean \pm standard deviation

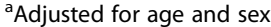

$C I$ confidence interval, $p K E M P-d B W$ percent knee extension muscle power to dry body weight, SPPB short physical performance battery 
future study. In addition, slow resistance training could be performed at a lower intensity with little influence on cardiovascular systems, maintaining the anabolic effect on the muscles [20].

\section{Impact on physical performance}

First of all, we employed SPPB, the 6-min walking test, and gait-speed as representative values describing patients' physical performance in this discussion section [19]. By this definition, there is conflicting evidence present regarding the impact of resistance training on physical performance in patients on MHD $[13-15,17,18]$. For example, Headley S and Chen JL et al. reported the positive impact of resistance training on physical performance after 12 weeks of training in their interventional study, with data from 10 patients in 2002, and after presumably 18 weeks of training (36 sessions, twice a week), with data from 50 patients in 2010 [13, 17]. However, three other studies did not report a significant change in physical performance after 12 weeks of training [14, 15, 18]. One of the possible mechanisms of this discrepancy is the short training period. In fact, all negative results were reported by trials which provided 3-month training and we speculate that a long-term training period is needed as the anabolic effect on muscle fibers may need more time in elderly patients [28]. The other possible mechanism for the discrepancy is the low statistical power due to the small number of patients who receive intervention ( $n \leq 25$ in all negative studies) $[14,15,18]$. Consequently, two important aspects of the present study included the large number of study population $(n=306)$ and 6 -month resistance training, providing physicians with clinically relevant insights. At any rate with our results, we speculate that resistance training has the potential to prevent the progression of sarcopenia and frailty in patients with MHD over a longer time period, improving QOL and survival. Further evaluation should be warranted for long-term efficacy.

\section{Clinical implication}

Although our study was observational, and as such we could not assess the impact of resistance training accurately, our findings provide physicians with complementary insights in the field of resistance exercise training for patients on MHD. Given that our study included a large number of patients with a long follow-up time, we provide additional evidence and validation in support of previous reports describing the short term favorable impact of resistance training, or those examining a variety of exercise training programs in patients on MHD [7-18].

\section{Study limitations}

Our study has several limitations that warrant mention. Firstly, we could not evaluate several risk factors that are associated with muscle wasting in patients with MHD, such as underlying disorders, hormonal alterations, inflammation, concurrent comorbidities or nutritional interventional information, due to the retrospective nature of this study $[6,12,15]$. However, in the present study, the nutritional status of the study population could be estimated by baseline n-PCR, albumin, or cholesterol levels (Table 1) [29, 30]. Secondly, we only included patients who had completed the 6-month training programs and our study has significant selection bias, although previous randomized controlled trials also had similar selection bias, with good adherence shown by the high completion rate of the study [13-18]. Third, we did not measure muscle mass in a daily clinical practice as previous studies have demonstrated that an improvement in muscle strength is attributable to the improvements in muscle mass [5, 24]. Thus, we could not collect data relating to muscle mass in this retrospective observational study. However, we did present data on the \%CGR as an index of muscle mass. Finally, it is possible that some of the study patients self-trained at home, as $44.2 \%$ had an exercise habit at baseline. However, considering the increasing interest in obtaining real-world observational data in clinical decision making as complementary evidence for those from randomized controlled trials, we believe the importance of our results overweight these limitations [31, 32].

\section{Conclusions}

Six-month resistance training was associated with improved muscle strength and physical performance in patients with MHD.

\section{Abbreviations}

\%CGR: Percent creatinine generation rate; HD: Hemodialysis;

MHD: Maintenance hemodialysis; n-PCR: Normalized protein catabolic rate; pKEMP-dBW: Percent knee extension muscle power to dry body weight;

QOL: Quality of life; SPPB: Short physical performance battery

\section{Acknowledgements}

We would like to express our heartfelt gratitude to the Japan Society of Clinical Research for their dedicated support.

\section{Funding}

The authors have no funding sources to declare.

\section{Availability of data and materials}

The datasets generated and/or analyzed during the current study are not publicly available but are available from the corresponding author on reasonable request.

\section{Authors' contributions}

YM, MH, SA, HI, KK, and MT contributed to the conception and design of the work, the acquisition, analysis and interpretation of data, drafting or revision of the manuscript, and final approval of the version to be submitted. 


\section{Ethics approval and consent to participate}

The study protocol complied with the Helsinki Declaration standards and was approved by the Ethical Committee of Nagoya Kyoritsu Hospital (Approval No. K094-03). The requirement of written informed consent was waived as this study used retrospective data.

\section{Consent for publication}

Written informed consent was not obtained for the publication of Fig. 1 because the image is entirely unidentifiable and there are no details on individuals reported within the manuscript.

\section{Competing interests}

The authors declare that they have no competing interests.

\section{Publisher's Note}

Springer Nature remains neutral with regard to jurisdictional claims in published maps and institutional affiliations.

\section{Author details}

'Department of Health Fitness Program, Nagoya Kyoritsu Hospital, Nagoya, Japan. ${ }^{2}$ Department of Clinical Investigation, Japan Society of Clinical Research, Kita-ku Umeda 1-11-1000, Osaka Ekimae 4th Bldg 10F, Osaka 530-0001, Japan. ${ }^{3}$ Center for Community-based Healthcare Research and Education, Shimane University, Izumo, Japan. ${ }^{4}$ Department of Nephrology, Nippon Medical School Graduate School of Medicine, Tokyo, Japan. ${ }^{5}$ Department of Nephrology, Nagoya First Red Cross Hospital, Aichi, Japan. ${ }^{6}$ Department of Physical Therapy, International University of Health and Welfare School of Health Science at Narita, Narita, Japan. ${ }^{7}$ Department of Minimally Invasive Surgical and Medical Oncology, Fukushima Medical University, Fukushima, Japan.

\section{Received: 20 January 2019 Accepted: 7 May 2019}

\section{Published online: 16 May 2019}

\section{References}

1. Obrador GT, Pereira BJ, Kausz AT. Chronic kidney disease in the United States: an underrecognized problem. Semin Nephrol. 2002;22:441-8.

2. Coresh J, Selvin E, Stevens LA, Manzi J, Kusek JW, Eggers P, Van Lente F, Levey AS. Prevalence of chronic kidney disease in the United States. JAMA 2007;298:2038-47

3. Kurella Tamura M, Covinsky KE, Chertow GM, Yaffe K, Landefeld CS, McCulloch CE. Functional status of elderly adults before and after initiation of dialysis. N Engl J Med. 2009;361:1539-47.

4. Noori N, Kopple JD, Kovesdy CP, Feroze U, Sim JJ, Murali SB, Luna A, Gomez M, Luna C, Bross R, Nissenson AR, Kalantar-Zadeh K. Mid-arm muscle circumference and quality of life and survival in maintenance hemodialysis patients. Clin J Am Soc Nephrol. 2010;5:2258-68.

5. Isoyama N, Qureshi AR, Avesani $C M$, Lindholm B, Bàràny $P$, Heimbürger $O$, Cederholm T, Stenvinkel P, Carrero JJ. Comparative associations of muscle mass and muscle strength with mortality in dialysis patients. Clin J Am Soc Nephrol. 2014;9:1720-8.

6. Beaudart C, Dawson A, Shaw SC, Harvey NC, Kanis JA, Binkley N, Reginster JY, Chapurlat R, Chan DC, Bruyère O, Rizzoli R, Cooper C, Dennison EM, IOFESCEO Sarcopenia Working Group. Nutrition and physical activity in the prevention and treatment of sarcopenia: systematic review. Osteoporos Int. 2017;28:1817-33.

7. Kopple JD, Wang H, Casaburi R, Fournier M, Lewis MI, Taylor W, Storer TW. Exercise in maintenance hemodialysis patients induces transcriptional changes in genes favoring anabolic muscle. J Am Soc Nephrol. 2007;18: 2975-86.

8. Cho JH, Lee JY, Lee S, Park H, Choi SW, Kim JC. Effect of intradialytic exercise on daily physical activity and sleep quality in maintenance hemodialysis patients. Int Urol Nephrol. 2018;50:745-54.

9. Chung YC, Yeh ML, Liu YM. Effects of intradialytic exercise on the physical function, depression and quality of life for haemodialysis patients: a systematic review and meta-analysis of randomised controlled trials. J Clin Nurs. 2017;26:1801-13.

10. Gomes Neto M, de Lacerda FFR, Lopes AA, Martinez BP, Saquetto MB. Intradialytic exercise training modalities on physical functioning and healthrelated quality of life in patients undergoing maintenance hemodialysis: systematic review and meta-analysis. Clin Rehabil. 2018;32:1189-202.
11. Ikizler TA. Exercise as an anabolic intervention in patients with endstage renal disease. J Ren Nutr. 2011;21:52-6.

12. Rhee CM, Kalantar-Zadeh K. Resistance exercise: an effective strategy to reverse muscle wasting in hemodialysis patients? J Cachexia Sarcopenia Muscle. 2014;5:177-80.

13. Headley S, Germain M, Mailloux P, Mulhern J, Ashworth B, Burris J, Brewer B, Nindl BC, Coughlin M, Welles R, Jones M. Resistance training improves strength and functional measures in patients with end-stage renal disease. Am J Kidney Dis. 2002:40:355-64.

14. Johansen KL, Painter PL, Sakkas GK, Gordon P, Doyle J, Shubert T. Effects of resistance exercise training and nandrolone decanoate on body composition and muscle function among patients who receive hemodialysis: a randomized, controlled trial. J Am Soc Nephrol. 2006;17: 2307-14.

15. Cheema B, Abas H, Smith B, O'Sullivan A, Chan M, Patwardhan A, Kelly J, Gillin A, Pang G, Lloyd B, Singh MF. Progressive exercise for anabolism in kidney disease (PEAK): a randomized, controlled trial of resistance training during hemodialysis. J Am Soc Nephrol. 2007;18:1594-601.

16. Cheema B, Abas H, Smith B, O'Sullivan A, Chan M, Patwardhan A, Kelly J, Gillin A, Pang G, Lloyd B, Fiatarone SM. Randomized controlled trial of intradialytic resistance training to target muscle wasting in ESRD: the progressive exercise for anabolism in kidney disease (PEAK) study. Am J Kidney Dis. 2007:50:574-84

17. Chen $J$, Godfrey S, Ng TT, Moorthi R, Liangos O, Ruthazer R, Jaber BL, Levey AS, Castaneda-Sceppa C. Effect of intra-dialytic, low-intensity strength training on functional capacity in adult haemodialysis patients: a randomized pilot trial. Nephrol Dial Transplant. 2010;25:1936-43.

18. Kirkman DL, Mullins P, Junglee NA, Kumwenda M, Jibani MM, Macdonald $J$ H. Anabolic exercise in haemodialysis patients: a randomised controlled pilot study. J Cachexia Sarcopenia Muscle. 2014;5:199-207.

19. Cruz-Jentoft AJ, Baeyens JP, Bauer JM, Boirie Y, Cederholm T, Landi F, Martin FC, Michel JP, Rolland Y, Schneider SM, Topinková E, Vandewoude M, Zamboni M, European working group on sarcopenia in older people. Sarcopenia: European consensus on definition and diagnosis: report of the European working group on sarcopenia in older people. Age Ageing. 2010; 39:412-23.

20. Watanabe $Y$, Madarame H, Ogasawara R, Nakazato K, Ishii N. Effect of very low-intensity resistance training with slow movement on muscle size and strength in healthy older adults. Clin Physiol Funct Imaging. 2014;34:463-70.

21. Borg GA. Psychophysical bases of perceived exertion. Med Sci Sports Exerc. 1982;14:377-81.

22. Katoh M. Reliability of isometric knee extension muscle strength measurements made by a hand-held dynamometer and a belt: a comparison of two types of device. J Phys Ther Sci. 2015;27:851-4.

23. Aoyama M, Ohnishi Y, Utsunomiya H, Kanezaki S, Takeuchi H, Watanuki M, Matsuda DK, Uchida S. A prospective, randomized, controlled trial comparing conservative treatment with trunk stabilization exercise to standard hip muscle exercise for treating femoroacetabular impingement: a pilot study. Clin J Sport Med. 2017 [Epub ahead of print]. https://doi.org/10. 1097/JSM.0000000000000516.

24. Nilwik R, Snijders T, Leenders M, Groen BB, van Kranenburg J, Verdijk LB, van Loon $L$. The decline in skeletal muscle mass with aging is mainly attributed to a reduction in type II muscle fiber size. Exp Gerontol. 2013;48:492-8.

25. Verdijk LB, Snijders T, Drost M, Delhaas T, Kadi F, van Loon LJ. Satellite cells in human skeletal muscle; from birth to old age. Age (Dordr). 2014;36:545-7.

26. McGinley SK, Armstrong MJ, Boulé NG, Sigal RJ. Effects of exercise training using resistance bands on glycaemic control and strength in type 2 diabetes mellitus: a meta-analysis of randomised controlled trials. Acta Diabetol. 2015;52:221-30.

27. Manfredini F, Mallamaci F, D'Arrigo G, Baggetta R, Bolignano D, Torino C, Lamberti N, Bertoli S, Ciurlino D, Rocca-Rey L, Barillà A, Battaglia Y, Rapanà RM, Zuccalà A, Bonanno G, Fatuzzo P, Rapisarda F, Rastelli S, Fabrizi F, Messa P, De Paola L, Lombardi L, Cupisti A, Fuiano G, Lucisano G, Summaria C, Felisatti M, Pozzato E, Malagoni AM, Castellino P, Aucella F, Abd ElHafeez S, Provenzano PF, Tripepi G, Catizone L, Zoccali C. Exercise in patients on dialysis: a multicenter, randomized clinical trial. J Am Soc Nephrol. 2017;28:1259-68.

28. Oesen S, Halper B, Hofmann M, Jandrasits W, Franzke B, Strasser EM, Graf A, Tschan H, Bachl N, Quittan M, Wagner KH, Wessner B. Effects of elastic band resistance training and nutritional supplementation on physical performance of institutionalised elderly--a randomized controlled trial. Exp Gerontol. 2015;72:99-108. 
29. Owen WF Jr, Lew NL, Liu Y, Lowrie EG, Lazarus JM. The urea reduction ratio and serum albumin concentration as predictors of mortality in patients undergoing hemodialysis. N Engl J Med. 1993;329:1001-6.

30. Goldwasser P, Mittman N, Antignani A, Burrell D, Michel MA, Collier J, Avram MM. Predictors of mortality in hemodialysis patients. J Am Soc Nephrol. 1993;3:1613-22.

31. Dal-Ré R, Janiaud P, loannidis JPA. Real-world evidence: how pragmatic are randomized controlled trials labeled as pragmatic? BMC Med. 2018;16:49.

32. Corrigan-Curay J, Sacks L, Woodcock J. Real-world evidence and real-world data for evaluating drug safety and effectiveness. JAMA. 2018;320:867-8.

Ready to submit your research? Choose BMC and benefit from:

- fast, convenient online submission

- thorough peer review by experienced researchers in your field

- rapid publication on acceptance

- support for research data, including large and complex data types

- gold Open Access which fosters wider collaboration and increased citations

- maximum visibility for your research: over $100 \mathrm{M}$ website views per year

At BMC, research is always in progress.

Learn more biomedcentral.com/submissions 